\title{
Integrated analysis of the potential roles of miRNA-mRNA networks in triple negative breast cancer
}

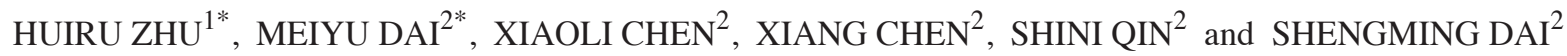 \\ ${ }^{1}$ Department of Galactophore, The Third Affiliated Hospital of Guangxi University of Chinese Medicine, Liuzhou, \\ Guangxi 545001; ${ }^{2}$ Department of Clinical Laboratory, The Fourth Affiliated Hospital \\ of Guangxi Medical University, Liuzhou, Guangxi 545005, P.R. China
}

Received June 14, 2016; Accepted April 12, 2017

DOI: $10.3892 / \mathrm{mmr} .2017 .6750$

\begin{abstract}
Triple negative breast cancer (TNBC) is a type of breast cancer where the tumor cells are negative for the estrogen, progesterone and human epidermal growth factor 2 receptors. To date, expression profiling of microRNA (miRNA/miR) and mRNA sequences have been widely applied for the diagnosis of TNBC. In the present study, an integrated analysis of miRNA-mRNA profiling arrays was performed. A total of five dysregulated miRNAs in patients with TNBC were identified, including upregulated miR-558 expression and downregulated miR-320d-1, miR-548v, miR-99a and miR-21 expression. In addition, 49 potential target mRNA sequences were identified. Bioinformatics analyses were performed on the identified miRNAs and mRNAs, including gene ontology (GO), Kyoto Encyclopedia of Genes and Genomes pathway and miRNA-mRNA network analyses. A total of $31 \mathrm{GO}$ terms and three signaling pathways were identified. The results indicated that the differentially expressed miRNAs and their potential target mRNAs may affect the pathogenesis of TNBC, and may therefore be considered as promising biomarkers for the early diagnosis and targeted therapy of patients with TNBC.
\end{abstract}

\section{Introduction}

Among all breast cancer types, 15-20\% are pathologically diagnosed as triple negative breast cancer (TNBC). A typical immunohistochemical feature of TNBC tumors is that they are negative for the estrogen, progesterone and human

Correspondence to: Professor Shengming Dai, Department of Clinical Laboratory, The Fourth Affiliated Hospital of Guangxi Medical University, 1 Liushi Road, Liuzhou, Guangxi 545005, P.R. China

E-mail: daishm@sina.com

${ }^{*}$ Contributed equally

Key words: triple negative breast cancer, microRNA, mRNA, gene ontology analysis, pathway analysis epidermal growth factor 2 receptors (1). TNBC is highly invasive with common symptoms including breast lumps and nipple discharge. Epidemiological studies indicate that TNBC generally develops among pre-menopausal women, and particularly among young African-American women (2). It has been reported that TNBC is frequently diagnosed at stage III, and is usually correlated with increased metastasis, disease recurrence and cancer cell proliferation and invasion (3). To date, no specific treatment guidelines have been developed for TNBC. At present, the common methods for the treatment of breast cancer include surgery, radiotherapy, chemotherapy and traditional Chinese medicine. Therefore, patients with TNBC are currently treated with methods common to all breast cancer types. However, the rapid development of metastases and high recurrence rates lead to poor patient prognoses and high mortality rates $(4,5)$.

MicroRNAs (miRNA/miR) are endogenous non-coding small RNA sequences that are 20-24 nucleotides in length. miRNAs regulate tumor cell metabolism, proliferation, invasion and metastasis (3). Each miRNA is able to regulate multiple target genes, while different miRNAs are able to regulate the same target gene. It is thought that approximately one-third of human genes are regulated by miRNAs (6). Previous studies indicate that $\sim 70 \%$ of miRNAs in mammalian cells are located in miRNA transcription units (7). In addition, the majority of miRNAs are located in introns, which are highly conserved in different species (8). The high conservation of miRNAs among different species suggests common and important functions. Therefore, investigating the mechanisms and functions of miRNAs may be useful for the diagnosis and treatment of human cancers. To date, a number of previous studies have attempted to elucidate the mechanisms and functions of miRNAs in the diagnosis and treatment of human cancer; however, only a limited number have focused on understanding the function of miRNAs in the pathogenesis of TNBC $(9,10)$. In addition, the mechanisms and signaling pathways regulated by miRNAs and their associated target genes require further investigation. With the development of gene expression microarrays and bioinformatics analysis technologies, an integrated and detailed investigation of the aberrant expression of miRNA and mRNA sequences in patients with TNBC was performed in the current study. The aim was to identify differentially expressed miRNA and 
mRNA sequences in TNBC tissues compared with paired normal adjacent tissues using published data from the Gene Expression Omnibus (GEO). In addition, bioinformatics analysis was performed to investigate the function and signaling pathways of the identified miRNA and target mRNA sequences.

\section{Materials and methods}

Microarray data. Two expression microarrays, which included miRNAs and mRNAs, were obtained from the GEO database (www.ncbi.nlm.nih.gov/geo). The accession numbers of these arrays were GSE61723 and GSE61724, which were based on the GPL16686 [HuGene-2_0-st, Affymetrix Human Gene 2.0 ST Array, transcript (gene) version] and GPL6244 [HuGene-1_0-st, Affymetrix Human Gene 1.0 ST Array, transcript (gene) version] platforms, respectively. The GSE61723 test dataset consisted of 33 TNBC tissue samples and 17 normal adjacent tissue samples. The GSE61724 validation dataset consisted of 16 TNBC tissue samples and 4 normal adjacent tissue samples. More detailed information regarding the tumor samples have been reported in a previous study (11).

Data processing and differential expression analysis. Series matrix files were employed and analyzed in the present study. As all microarray data consisted of preprocessed normalized data, a fold-change of $\geq 2.0$ and a p-value of $\leq 0.05$ were used as the thresholds for screening differentially-expressed miRNAs and mRNAs, using $\mathrm{R}$ software (version 3.3.2; www.r-project.org) and the edgeR package. Hierarchical clustering analysis was performed using Multiple Experiment Viewer software (version, 4.90; www.mev.tm4.org), and the Pearson correlation distance metric and average linkage method.

Prediction of miRNA target genes. The target genes of differentially expressed miRNAs were predicted using TargetScan software (version 7.0; www.targetscan.org) (12). In order to reduce the probability of identifying incorrect target genes, potential target mRNAs were selected using the intersection set for the differentially expressed mRNAs identified from the GSE61723 dataset, and the predicted target genes were identified using TargetScan software. The intersection set signifies that the genes existed in multiple data sets. The miRNA-mRNA interaction regulatory network was constructed using Cytoscape software (version 3.3.0; www. cytoscape.org) (13).

Gene ontology (GO) and Kyoto Encyclopedia of Genes and Genomes (KEGG) pathway analysis. Enrichment analyses for GO processes and KEGG signaling pathways were performed using the Database for Annotation, Visualization, and Integrated Discovery (DAVID; version 6.8) software program (www.david.ncifcrf.gov) $(14,15)$. Biological processes, cellular components and molecular functions were the main GO terms used for functional analysis of the potential targets of miRNAs (16). GO terms and KEGG pathways were selected using a threshold of $\mathrm{P} \leq 0.05$ and a count of $\geq 2$.
Table I. miRNAs displaying significantly altered expression levels in triple negative breast cancer samples from GSE61723.

A, Downregulated

\begin{tabular}{lcc}
\hline miRNA & Fold change & P-value \\
\hline hsa-miR-320d-1 & -2.78 & $9.66 \times 10^{-4}$ \\
hsa-miR-548v & -2.28 & $3.80 \times 10^{-3}$ \\
hsa-miR-99a & -2.22 & $1.41 \times 10^{-6}$ \\
hsa-miR-21 & -2.07 & $6.26 \times 10^{-4}$ \\
\hline
\end{tabular}

B, Upregulated

hsa-miR-558

2.01

$1.47 \times 10^{-3}$

miRNA/miR, microRNA.

Table II. mRNAs displaying significantly altered expression patterns in triple negative breast cancer samples from GSE61723.

A, Upregulated

\begin{tabular}{lcc}
\hline mRNA & Fold change & P-value \\
\hline SKA3 & 2.00 & $8.80 \times 10^{-6}$ \\
PRDX1 & 2.00 & $2.67 \times 10^{-5}$ \\
DEK & 2.01 & $2.76 \times 10^{-4}$ \\
SMC4 & 2.01 & $1.56 \times 10^{-5}$ \\
SYNGR2 & 2.02 & $7.77 \times 10^{-4}$ \\
SKIL & 2.02 & $9.45 \times 10^{-5}$ \\
RARRES1 & 2.02 & $9.01 \times 10^{-3}$ \\
CXCL11 & 2.03 & $6.14 \times 10^{-5}$ \\
GFPT1 & 2.03 & $1.74 \times 10^{-4}$ \\
LRRC15 & 2.04 & $1.22 \times 10^{-4}$ \\
C21orf91 & 2.05 & $4.29 \times 10^{-5}$ \\
SNRPD1 & 2.06 & $1.18 \times 10^{-4}$ \\
DSC3 & 2.10 & $6.24 \times 10^{-3}$ \\
MCUR1 & 2.11 & $1.89 \times 10^{-4}$ \\
SRP9 & 2.11 & $4.66 \times 10^{-4}$ \\
CHML & 2.12 & $2.98 \times 10^{-5}$ \\
ARNTL2 & 2.12 & $2.27 \times 10^{-4}$ \\
STRAP & 2.13 & $3.37 \times 10^{-5}$ \\
MELK & 2.13 & $2.71 \times 10^{-5}$ \\
STIL & 2.14 & $7.21 \times 10^{-6}$ \\
NUSAP1 & 2.14 & $7.03 \times 10^{-7}$ \\
TYMS & 2.15 & $4.99 \times 10^{-3}$ \\
GLYATL2 & 2.15 & $9.02 \times 10^{-3}$ \\
HMGA1 & 2.17 & $4.52 \times 10^{-4}$ \\
MKI67 & 2.18 & $9.96 \times 10^{-6}$ \\
UBE2T & 2.18 & $2.82 \times 10^{-5}$ \\
CALU & 2.19 & $3.88 \times 10^{-6}$ \\
RAD51AP1 & 2.20 & $7.80 \times 10^{-6}$ \\
RGS1 & $1.13 \times 10^{-4}$ \\
RBM34 & 2.20 & $7.94 \times 10^{-7}$ \\
& 2.21 & \\
\hline
\end{tabular}


Table II. Continued.

A, Upregulated

\begin{tabular}{|c|c|c|}
\hline mRNA & Fold change & $\mathrm{P}$-value \\
\hline GPI & 2.23 & $9.43 \times 10^{-6}$ \\
\hline ESRP1 & 2.23 & $8.75 \times 10^{-6}$ \\
\hline MYBL1 & 2.24 & $7.28 \times 10^{-4}$ \\
\hline STK38L & 2.25 & $3.86 \times 10^{-6}$ \\
\hline PRR11 & 2.30 & $1.58 \times 10^{-6}$ \\
\hline AMD1 & 2.32 & $3.92 \times 10^{-4}$ \\
\hline DSC2 & 2.38 & $9.00 \times 10^{-4}$ \\
\hline KIF11 & 2.40 & $1.92 \times 10^{-6}$ \\
\hline NDC80 & 2.42 & $8.30 \times 10^{-7}$ \\
\hline TMSB15A & 2.48 & $8.05 \times 10^{-4}$ \\
\hline BGN & 2.48 & $1.21 \times 10^{-5}$ \\
\hline MT1H & 2.51 & $7.29 \times 10^{-3}$ \\
\hline ECT2 & 2.52 & $4.48 \times 10^{-7}$ \\
\hline CCNA2 & 2.55 & $1.79 \times 10^{-6}$ \\
\hline S100A9 & 2.76 & $9.01 \times 10^{-4}$ \\
\hline LYZ & 2.77 & $1.03 \times 10^{-3}$ \\
\hline TMEM65 & 2.79 & $7.64 \times 10^{-5}$ \\
\hline CD24 & 3.24 & $1.45 \times 10^{-5}$ \\
\hline HORMAD1 & 3.28 & $4.46 \times 10^{-6}$ \\
\hline TMSB10 & 3.31 & $1.09 \times 10^{-8}$ \\
\hline SULF1 & 3.38 & $7.50 \times 10^{-8}$ \\
\hline SPP1 & 3.40 & $2.30 \times 10^{-6}$ \\
\hline FN1 & 3.94 & $1.26 \times 10^{-6}$ \\
\hline TOP2A & 4.13 & $6.26 \times 10^{-8}$ \\
\hline CKS2 & 4.20 & $6.45 \times 10^{-8}$ \\
\hline CXCL10 & 4.48 & $1.19 \times 10^{-5}$ \\
\hline CXCL9 & 5.14 & $1.96 \times 10^{-5}$ \\
\hline LOC $100507381^{\mathrm{a}}$ & 2.16 & $4.06 \times 10^{-4}$ \\
\hline $\mathrm{UBE}^{2} \mathrm{C}^{\mathrm{a}}$ & 2.22 & $1.53 \times 10^{-7}$ \\
\hline
\end{tabular}

B, Downregulated

\begin{tabular}{lll}
\hline PIP & -8.96 & $1.08 \times 10^{-6}$ \\
APOD & -7.77 & $9.23 \times 10^{-9}$ \\
ANKRD30A & -4.91 & $9.08 \times 10^{-12}$ \\
OGN & -4.13 & $7.52 \times 10^{-11}$ \\
DCN & -3.29 & $8.40 \times 10^{-10}$ \\
PIGR & -3.19 & $5.37 \times 10^{-7}$ \\
MUCL1 & -3.17 & $1.34 \times 10^{-3}$ \\
EGR1 & -3.09 & $3.01 \times 10^{-8}$ \\
AGR3 & -3.01 & $8.72 \times 10^{-12}$ \\
IGF1 & -2.96 & $1.21 \times 10^{-8}$ \\
CPE & -2.74 & $6.18 \times 10^{-9}$ \\
LIFR & -2.67 & $3.25 \times 10^{-7}$ \\
SPARCL1 & -2.65 & $9.09 \times 10^{-6}$ \\
CXCL12 & -2.60 & $8.37 \times 10^{-9}$ \\
LEP & -2.53 & $1.77 \times 10^{-6}$ \\
FGF7 & -2.53 & $1.47 \times 10^{-4}$ \\
CD36 & -2.48 & $4.12 \times 10^{-7}$ \\
DCLK1 & -2.46 & $1.27 \times 10^{-8}$
\end{tabular}

Table II. Continued.

B, Downregulated

\begin{tabular}{lcc}
\hline mRNA & Fold change & P-value \\
\hline FGF10 & -2.34 & $7.78 \times 10^{-11}$ \\
PCDH18 & -2.29 & $6.78 \times 10^{-6}$ \\
TSHZ2 & -2.28 & $2.10 \times 10^{-6}$ \\
TAT & -2.27 & $6.83 \times 10^{-6}$ \\
ANKRD30B & -2.24 & $4.58 \times 10^{-4}$ \\
CHRDL1 & -2.21 & $3.71 \times 10^{-7}$ \\
FREM1 & -2.18 & $9.51 \times 10^{-9}$ \\
CCL28 & -2.14 & $9.17 \times 10^{-5}$ \\
KIT & -2.14 & $1.47 \times 10^{-2}$ \\
EFEMP1 & -2.13 & $2.58 \times 10^{-4}$ \\
AGR2 & -2.10 & $3.56 \times 10^{-6}$ \\
JAM2 & -2.08 & $6.94 \times 10^{-8}$ \\
MME & -2.08 & $6.00 \times 10^{-5}$ \\
CD34 & -2.07 & $1.14 \times 10^{-6}$ \\
PI15 & -2.07 & $1.23 \times 10^{-4}$ \\
AREG & -2.06 & $2.80 \times 10^{-6}$ \\
AADACL2 & -2.06 & $3.20 \times 10^{-6}$ \\
KCTD14 & -2.05 & $4.39 \times 10^{-3}$ \\
TMEM144 & -2.04 & $5.22 \times 10^{-7}$ \\
C3orf62 & -2.02 & $4.69 \times 10^{-6}$ \\
& & \\
\hline
\end{tabular}

${ }^{\mathrm{a}}$ Genes which have been deleted from the NCBI database.

\section{Results}

Identification of differentially expressed miRNAs and mRNAs. In order to gain an improved understanding of the regulatory mechanisms underlying miRNA-mRNA interactions in TNBC, comprehensive microarray expression profiles were employed to identify differentially expressed miRNAs and mRNAs. By comparing TNBC tissues with normal adjacent tissues, five miRNAs (upregulated miR-558 and downregulated miR-320d-1, miR-548v, miR-99a and miR-21 expression) and 97 mRNAs were identified with a threshold of $\mathrm{P} \leq 0.05$ and a fold-change of $\geq 2.0$ (Tables I and II). According to the GenBank database (www.ncbi.nlm.nih.gov/genbank), LOC100507381 was removed due to inconsistencies with standard genome annotation processing. In addition, the gene UBE2C was permanently removed from the NCBI database, as there is not enough evidence to prove its presence in subsequent studies. Therefore, 95 differentially expressed mRNAs were identified between the 33 TNBC tissue and 17 normal adjacent tissue samples. Unsupervised hierarchical clustering analyses were performed using a fold-change threshold of $\geq 2.0$. The results identified five differentially expressed miRNAs and 95 differentially expressed mRNAs (Figs. 1 and 2).

Validation of miRNA expression. To confirm the five differentially expressed miRNAs identified in TNBC samples from the GSE61723 array, microarray data from the GSE61724 array was obtained from the GEO database, which included 


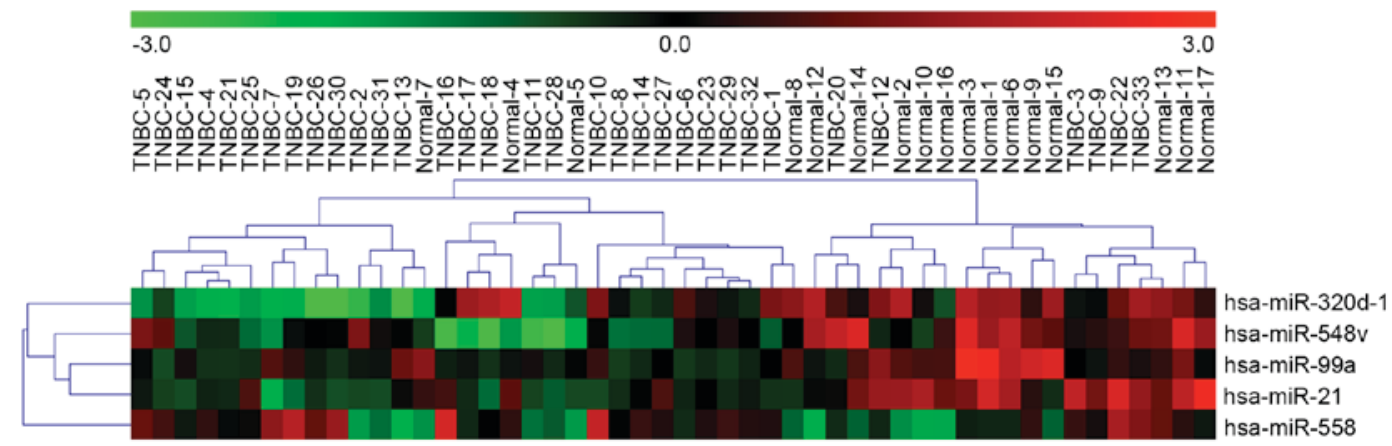

Figure 1. Unsupervised hierarchical clustering analysis of TNBC tissues and normal adjacent tissues from the GSE61723 array identified five differentially expressed miRNAs. TNBC, triple negative breast cancer; miR, microRNA.

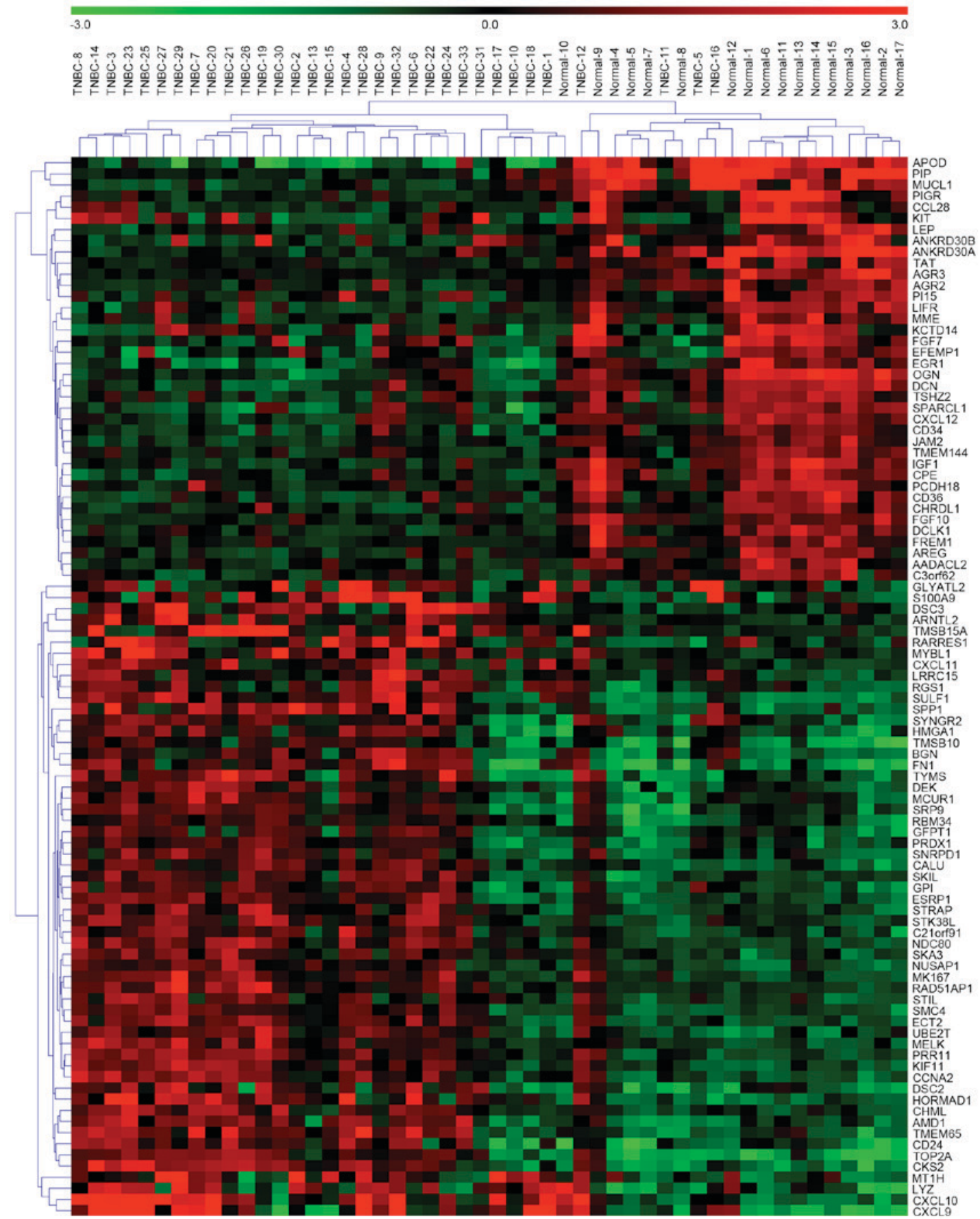

Figure 2. Unsupervised hierarchical clustering analysis of TNBC tissues and normal adjacent tissues from the GSE61723 array identified 95 differentially expressed mRNAs. TNBC, triple negative breast cancer. 


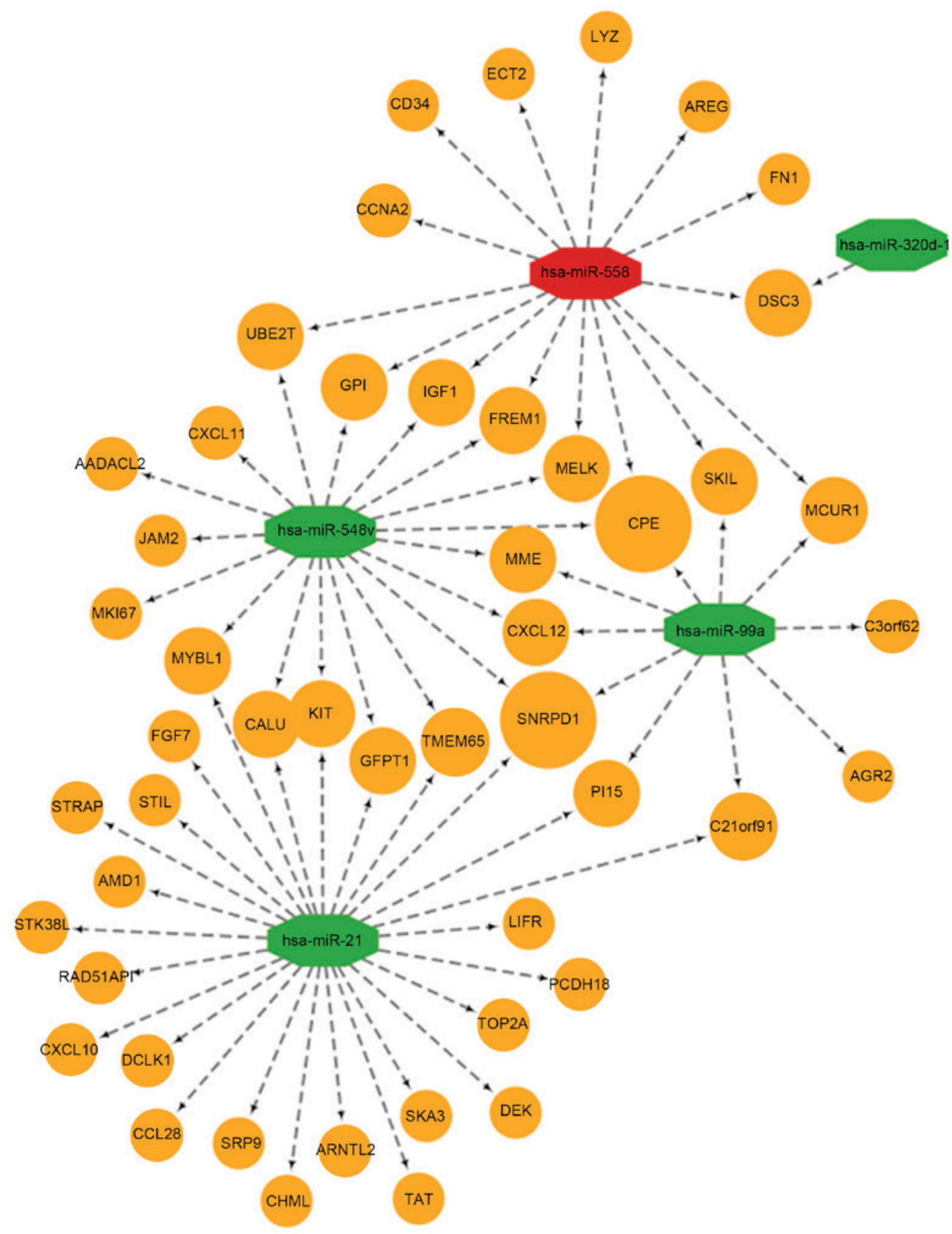

Figure 3. miRNA-mRNA interaction regulatory network. The green color indicates downregulation and the red color indicates upregulation of miRNAs. Circular nodes represent mRNA, and the size of each node is proportional to the assumed functional connectivity of each miRNA according to the predicted target gene counts. miRNA, microRNA.

16 TNBC tissues and 4 normal adjacent tissue samples. Two miRNAs (miRNA-99a and miRNA-21) were selected for further validation from the five significantly dysregulated miRNAs. miRNA-99a $\left(\mathrm{P}=1.91 \times 10^{-6}\right)$ and miRNA-21 $\left(\mathrm{P}=1.84 \times 10^{-4}\right)$ were observed to be differentially expressed in TNBC samples when compared with normal adjacent samples in the GSE61723 array. Therefore, the results were consistent with the miRNA expression levels in the GSE61723 dataset, which demonstrated that the five dysregulated miRNAs were able to accurately distinguish between TNBC tissues and normal tissues. Data from GSE61723 and GSE61724 are not identical and certain miRNAs in GSE61723 were unable to be found in GSE61724; therefore, validation was only performed in two miRNAs.
Prediction of miRNA target genes and functional analysis. In order to determine the putative functions and target genes of the five dysregulated miRNAs identified in TNBC samples, the intersection set of sequences from the 95 differentially expressed mRNAs and the predicted target genes were identified using TargetScan software. A total of 49 target mRNAs were included for further analysis. The of miRNA-mRNA interaction regulatory network is shown in Fig. 3. The results indicated that miRNA-320d-1 targeted the smallest number of genes, whereas the remaining four miRNAs targeted at least 10 (Fig. 3). In addition, carboxypeptidase and the small nuclear ribonucleoprotein D1 polypeptide were identified as the hub nodes connected to three miRNAs, which suggests that they may serve a key role in cancer pathogenesis (Fig. 3). 
Table III. Significantly enriched GO processes and KEGG pathways with $\mathrm{P} \leq 0.05$ and a gene count of $\geq 2$.

A, GO processes

\begin{tabular}{|c|c|c|c|}
\hline Category & Function & Gene count & P-value \\
\hline GO:0005576 & Extracellular region $(\mathrm{CC})$ & 18 & $2.14 \times 10^{-5}$ \\
\hline GO:0005615 & Extracellular space (CC) & 11 & $2.20 \times 10^{-5}$ \\
\hline GO:0044421 & Extracellular region part $(\mathrm{CC})$ & 12 & $7.38 \times 10^{-5}$ \\
\hline GO:0005125 & Cytokine activity $(\mathrm{MF})$ & 6 & $2.44 \times 10^{-4}$ \\
\hline GO:0008009 & Chemokine activity (MF) & 4 & $3.22 \times 10^{-4}$ \\
\hline GO:0042379 & Chemokine receptor binding (MF) & 4 & $3.88 \times 10^{-4}$ \\
\hline GO:0008083 & Growth factor activity (MF) & 5 & $1.21 \times 10^{-3}$ \\
\hline GO:0030335 & Positive regulation of cell migration (BP) & 4 & $2.45 \times 10^{-3}$ \\
\hline GO:0040017 & Positive regulation of locomotion (BP) & 4 & $3.22 \times 10^{-3}$ \\
\hline GO:0051272 & Positive regulation of cell motion (BP) & 4 & $3.22 \times 10^{-3}$ \\
\hline GO:0008284 & Positive regulation of cell proliferation (BP) & 6 & $7.93 \times 10^{-3}$ \\
\hline GO:0016477 & Cell migration $(\mathrm{BP})$ & 5 & $9.49 \times 10^{-3}$ \\
\hline GO:0008283 & Cell proliferation (BP) & 6 & $9.80 \times 10^{-3}$ \\
\hline GO:0042330 & Taxis $(\mathrm{BP})$ & 4 & $1.25 \times 10^{-2}$ \\
\hline GO:0006935 & Chemotaxis (BP) & 4 & $1.25 \times 10^{-2}$ \\
\hline GO:0051674 & Localization of cell (BP) & 5 & $1.36 \times 10^{-2}$ \\
\hline GO:0048870 & Cell motility (BP) & 5 & $1.36 \times 10^{-2}$ \\
\hline GO:0006928 & Cell motion $(\mathrm{BP})$ & 6 & $1.38 \times 10^{-2}$ \\
\hline GO:0030334 & Regulation of cell migration (BP) & 4 & $1.44 \times 10^{-2}$ \\
\hline GO:0007155 & Cell adhesion $(\mathrm{BP})$ & 7 & $1.80 \times 10^{-2}$ \\
\hline GO:0022610 & Biological adhesion (BP) & 7 & $1.81 \times 10^{-2}$ \\
\hline GO:0031099 & Regeneration $(\mathrm{BP})$ & 3 & $1.85 \times 10^{-2}$ \\
\hline GO:0030246 & Carbohydrate binding (MF) & 5 & $1.93 \times 10^{-2}$ \\
\hline GO:0040012 & Regulation of locomotion (BP) & 4 & $2.02 \times 10^{-2}$ \\
\hline GO:0051270 & Regulation of cell motion (BP) & 4 & $2.05 \times 10^{-2}$ \\
\hline GO:0009611 & Response to wounding (BP) & 6 & $2.12 \times 10^{-2}$ \\
\hline GO:0000902 & Cell morphogenesis (BP) & 5 & $2.22 \times 10^{-2}$ \\
\hline GO:0035019 & Somatic stem cell maintenance (BP) & 2 & $2.70 \times 10^{-2}$ \\
\hline GO:0032989 & Cellular component morphogenesis (BP) & 5 & $3.15 \times 10^{-2}$ \\
\hline GO:0008354 & Germ cell migration $(\mathrm{BP})$ & 2 & $3.58 \times 10^{-2}$ \\
\hline GO:0007626 & Locomotory behavior (BP) & 4 & $4.98 \times 10^{-2}$ \\
\hline
\end{tabular}

B, KEGG pathways

\begin{tabular}{llll}
\hline KEGG: 04060 & Cytokine-cytokine receptor interaction & 6 & $2.86 \times 10^{-2}$ \\
KEGG: 04062 & Chemokine signaling pathway & 4 & $3.52 \times 10^{-2}$ \\
KEGG: 04640 & Hematopoietic cell lineage & 3 & $4.41 \times 10^{-2}$ \\
\hline
\end{tabular}

GO, gene ontology; KEGG, Kyoto Encyclopedia of Genes and Genomes; CC, cellular components; BP, biological processes; MF, molecular functions.

Analysis of the GO processes and KEGG pathways involving the intersectional 49 target genes was performed using the DAVID tool, with thresholds of $\geq 2$ gene counts and P-values of $\leq 0.05$. The results identified $31 \mathrm{GO}$ terms and three signaling pathways (Table III). The 10 most significant GO terms of the predicted target genes are indicated in Fig. 4. According to the biological function analysis of GO processes, a large proportion of the predicted targets of differentially expressed
miRNAs encode cellular components in extracellular regions, and are involved in cell migration, proliferation, motion, adhesion and regeneration (Table III and Fig. 4). These biological functions are considered to be crucial for human tumorigenesis and metastasis (17). In addition, KEGG pathway analysis demonstrated that cytokine-cytokine receptor interactions, chemokine signaling and hematopoietic cell lineage pathways, which are associated with cell growth, proliferation and 


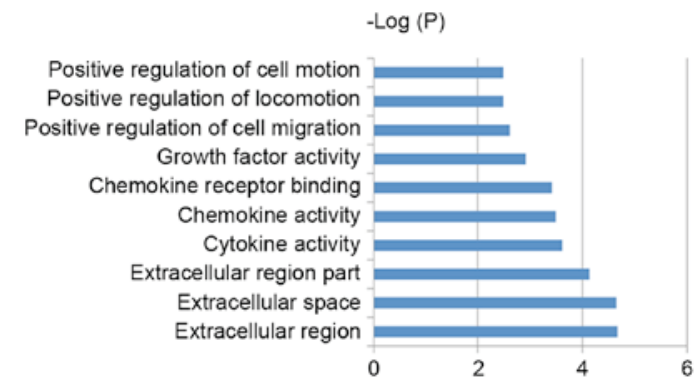

Figure 4. Ten most significant gene ontology terms of predicted microRNA target genes.

transformation, might be regulated by the predicted target genes (Table III).

\section{Discussion}

The mortality rates of patients with TNBC are high, as the vast majority of TNBC tumors are infiltrating ductal carcinomas, which often lead to visceral metastases (18). TNBC displays characteristic biological and clinical pathologic features, including rapid progression, poor prognosis and high recurrence rates (19). Surgery and chemotherapy are the major therapeutic strategies used to treat patients with TNBC. However, few studies have investigated the pathogenesis of TNBC. Therefore, there is an urgent need to identify novel therapeutic targets and treatments for patients with TNBC.

Expression microarrays have been widely applied for the diagnosis of various cancers $(20,21)$. An important application of microarray experiments is the analysis of differentially expressed miRNAs or mRNAs between normal and tumor samples. The differentially expressed miRNA or mRNA sequences may be used for further functional, pathway or bioinformatics analyses. Microarray analysis is generally expensive; however, it produces a large quantity of data. Therefore, integrated mining of microarray data from public databases, such as the GEO database, is popular among researchers (22-24).

In the present study, five differentially expressed miRNAs (upregulated miR-558 and downregulated miR-320d-1, miR-548v, miR-99a and miR-21 expression), and 97 mRNAs were identified in TNBC tissue samples when compared with normal adjacent tissues using the GSE61723 array. GSE61724 microarray data was subsequently used to validate the five differentially expressed miRNAs, and the results demonstrated that two of the identified miRNA sequences (miRNA-99a and miRNA-21) were differentially expressed in the GSE61724 dataset. A total of 49 predicted target mRNAs of the 5 miRNAs were selected for further analysis according to the intersection set of the 97 differentially expressed mRNAs and the predicted target genes using TargetScan software. In addition, bioinformatics analyses were employed to investigate the functions and signaling pathways of the miRNA-mRNA network, in order to further investigate the possible mechanisms underlying the development of TNBC. This may provide relevant targets for the early diagnosis and treatment of patients with TNBC.

$\mathrm{GO}$ analysis is divided into three sections (molecular function, biological process and cellular component), which are widely used in the organizational and functional annotation of genes (25). Previous studies have primarily focused on applying GO analysis for miRNA or mRNA microarray results (26-28). In the current study, 31 GO terms were identified using the DAVID software tool with threshold values of $\geq 2$ counts and $\mathrm{P} \leq 0.05$. The results revealed that the predicted target genes encode regulatory factors and proteins located in extracellular regions, and may be involved in a number of biological processes, including regulation of cell migration, cell motion, cell proliferation, and cell adhesion. These processes are closely associated with the occurrence and metastasis of human tumor cells (29).

Signaling pathways are the way of facilitating the effect of extracellular signaling molecules within cells. In the present study, three main signaling pathways were demonstrated to be regulated by 49 target genes of the five differentially expressed miRNAs. These pathways included cytokine-cytokine receptor interaction, chemokine and hematopoietic cell lineage pathways. This suggests that these pathways may serve a significant role in tumor progression, prevention and survival rates (30-32). These identified signaling pathways, may provide promising novel therapeutic targets for the treatment of patients with TNBC.

The present study has a number of limitations. Firstly, due to the lack of microarray data comparing TNBC tumor tissues with normal adjacent control tissues, the results of only two GSE series were employed in the current study. Secondly, due to the small number of differentially expressed miRNAs identified, analysis of the associated functions and signaling pathways may be incomplete. In future studies, a more comprehensive analysis will be performed using a greater number of microarray datasets.

In conclusion, five differentially expressed miRNAs and 49 potential target genes were identified in TNBC samples when compared with normal adjacent control tissues. The results of integrated $\mathrm{GO}$ function and KEGG signaling pathway analyses suggested that the differentially expressed miRNAs and their potential target mRNAs may influence the pathogenesis of TNBC. In addition, the results suggest that these sequences may be promising biomarkers for the early diagnosis and targeted therapy of patients with TNBC.

\section{References}

1. Perou CM, Sørlie T, Eisen MB, van de Rijn M, Jeffrey SS, Rees CA, Pollack JR, Ross DT, Johnsen H, Akslen LA, et al: Molecular portraits of human breast tumours. Nature 406: 747-752, 2000.

2. Pierobon $M$ and Frankenfeld CL: Obesity as a risk factor for triple-negative breast cancers: A systematic review and meta-analysis. Breast Cancer Res Treat 137: 307-314, 2013.

3. Foulkes WD, Smith IE and Reis-Filho JS: Triple-negative breast cancer. N Engl J Med 363: 1938-1948, 2010.

4. Lehmann BD, Bauer JA, Chen X, Sanders ME, Chakravarthy AB, Shyr Y and Pietenpol JA: Identification of human triple-negative breast cancer subtypes and preclinical models for selection of targeted therapies. J Clin Invest 121: 2750-2767, 2011.

5. Blows FM, Driver KE, Schmidt MK, Broeks A, van Leeuwen FE, Wesseling J, Cheang MC, Gelmon K, Nielsen TO, Blomqvist C, et al: Subtyping of breast cancer by immunohistochemistry to investigate a relationship between subtype and short and long term survival: A collaborative analysis of data for 10,159 cases from 12 studies. PLoS Med 7: e1000279, 2010.

6. Cui Q, Yu Z, Pan Y, Purisima EO and Wang E: MicroRNAs preferentially target the genes with high transcriptional regulation complexity. Biochem Biophys Res Commun 352: 733-738, 2007. 
7. Kim VN and Nam JW: Genomics of microRNA. Trends Genet 22: 165-173, 2006.

8. Rodriguez A, Griffiths-Jones S, Ashurst JL and Bradley A: Identification of mammalian microRNA host genes and transcription units. Genome Res 14: 1902-1910, 2004.

9. Yang L, Feng Y, Qi P, Xu S and Zhou Y: Mechanism of serum miR-21 in the pathogenesis of familial and triple negative breast cancer. J Biol Regul Homeost Agents 30: 1041-1045, 2016.

10. Zhang G, Liu Z, Han Y, Wang X and Yang Z: Overexpression of miR-509 increases apoptosis and inhibits invasion via suppression of tumor necrosis factor- $\alpha$ in triple-negative breast cancer Hs578T cells. Oncol Res 24: 233-238, 2016.

11. Mathe A, Wong-Brown M, Morten B, Forbes JF, Braye SG, Avery-Kiejda KA and Scott RJ: Novel genes associated with lymph node metastasis in triple negative breast cancer. Sci Rep 5: 15832,2015 .

12. Agarwal V, Bell GW, Nam JW and Bartel DP: Predicting effective microRNA target sites in mammalian mRNAs. Elife 4, 2015

13. Shannon P, Markiel A, Ozier O, Baliga NS, Wang JT, Ramage D, Amin N, Schwikowski B and Ideker T: Cytoscape: A software environment for integrated models of biomolecular interaction networks. Genome Res 13: 2498-2504, 2003

14. Huang da W, Sherman BT and Lempicki RA: Systematic and integrative analysis of large gene lists using DAVID bioinformatics resources. Nat Protoc 4: 44-57, 2009.

15. Huang da W, Sherman BT and Lempicki RA: Bioinformatics enrichment tools: Paths toward the comprehensive functional analysis of large gene lists. Nucleic Acids Res 37: 1-13, 2009.

16. Sam LT, Mendonca EA, Li J, Blake J, Friedman C and Lussier YA: PhenoGO: An integrated resource for the multiscale mining of clinical and biological data. BMC bioinformatics 10 (Suppl 2): S8, 2009

17. Labi V and Erlacher M: How cell death shapes cancer. Cell Death Dis 6: e1675, 2015

18. Zhang C, Wang S, Israel HP, Yan SX, Horowitz DP, Crockford S, Gidea-Addeo D, Clifford Chao KS, Kalinsky K and Connolly EP: Higher locoregional recurrence rate for triple-negative breast cancer following neoadjuvant chemotherapy, surgery and radiotherapy. Springerplus 4: 386, 2015.

19. Kim S, Park HS, Kim JY, Ryu J, Park S and Kim SI: Comparisons of oncologic outcomes between triple-negative breast cancer (TNBC) and Non-TNBC among patients treated with breast-conserving therapy. Yonsei Med J 57: 1192-1198, 2016.

20. Shi KQ, Lin, Z Chen XJ, Song M, Wang YQ, Cai YJ, Yang NB, Zheng MH, Dong JZ, Zhang L and Chen YP: Hepatocellular carcinoma associated microRNA expression signature: Integrated bioinformatics analysis, experimental validation and clinical significance. Oncotarget 6: 25093-25108, 2015.

21. Dai M, Chen X, Mo S, Li J, Huang Z, Huang S, Xu J, He B Zou Y, Chen J and Dai S: Meta-signature LncRNAs serve as novel biomarkers for colorectal cancer: Integrated bioinformatics analysis, experimental validation and diagnostic evaluation. Sci Rep 7: 46572, 2017.
22. An N, Shi X, Zhang Y, Lv N, Feng L, Di X, Han N, Wang G, Cheng $S$ and Zhang K: Discovery of a novel immune gene signature with profound prognostic value in colorectal cancer: A model of cooperativity disorientation created in the process from development to cancer. PloS One 10: e0137171, 2015.

23. Volinia S and Croce CM: Prognostic microRNA/mRNA signature from the integrated analysis of patients with invasive breast cancer. Proc Natl Acad Sci USA 110: 7413-7417, 2013

24. Zhang X, Peng Y, Jin Z, Huang W, Cheng Y, Liu Y, Feng X, Yang M, Huang Y, Zhao Z, et al: Integrated miRNA profiling and bioinformatics analyses reveal potential causative miRNAs in gastric adenocarcinoma. Oncotarget 6: 32878-32889, 2015.

25. Lovering RC, Camon EB, Blake JA and Diehl AD: Access to immunology through the gene ontology. Immunology 125 : $154-160,2008$

26. Xiong H, Li Q, Liu S, Wang F, Xiong Z, Chen J, Chen H, Yang Y, Tan X, Luo Q, et al: Integrated microRNA and mRNA transcriptome sequencing reveals the potential roles of miRNAs in stage I endometrioid endometrial carcinoma. PLoS One 9: e110163, 2014.

27. Li C, Nong Q, Solanki MK, Liang Q, Xie J, Liu X, Li Y, Wang W, Yang L and Li Y: Differential expression profiles and pathways of genes in sugarcane leaf at elongation stage in response to drought stress. Sci Rep 6: 25698, 2016.

28. Ma G, Luo Y, Zhu H, Luo Y, Korhonen PK, Young ND, Gasser RB and Zhou R: MicroRNAs of Toxocara canis and their predicted functional roles. Parasit Vectors 9: 229, 2016.

29. Ha M and Kim VN: Regulation of microRNA biogenesis. Nat Rev Mol Cell Biol 15: 509-524, 2014.

30. Lapkina-Gendler L, Rotem I, Pasmanik-Chor M, Gurwitz D, Sarfstein R, Laron Z and Werner H: Identification of signaling pathways associated with cancer protection in Laron syndrome. Endocr Relat Cancer 23: 399-410, 2016.

31. Shi KQ, Lin Z, Chen XJ, Song M, Wang YQ, Cai YJ, Yang NB, Zheng MH, Dong JZ, Zhang L and Chen YP: Hepatocellular carcinoma associated microRNA expression signature: Integrated bioinformatics analysis, experimental validation and clinical significance. Oncotarget 6: 25093-25108, 2015.

32. Geraldo MV and Kimura ET: Integrated analysis of thyroid cancer public datasets reveals role of post-transcriptional regulation on tumor progression by targeting of immune system mediators. PLoS One 10: e0141726, 2015. 